\title{
On Binomial Sums and Alternating Binomial Sums of Generalized Fibonacci Numbers with Falling Factorials
}

\author{
Sibel Koparal* and Neşe Ömür
}

\begin{abstract}
In this paper, we consider and obtain binomial sums and alternating binomial sums including falling factorial of the summation indice. For example, For integers $n$ and $m$ such that $0 \leq m<n$,

$$
\sum_{k=0}^{n}\left(\begin{array}{l}
n \\
k
\end{array}\right) k^{\underline{m}} U_{2 k}^{2 m}=\frac{n^{\underline{m}}}{\left(p^{2}+4\right)^{m}}\left(\sum_{i=0}^{m}(-1)^{i}\left(\begin{array}{c}
2 m \\
i
\end{array}\right) V_{2(m-i)}^{n-m} V_{2(m+n)(m-i)}-(-1)^{m} 2^{n-m}\left(\begin{array}{c}
2 m \\
m
\end{array}\right)\right),
$$

and for positive odd integer $m$,

$$
\sum_{k=0}^{n}(-1)^{k}\left(\begin{array}{l}
n \\
k
\end{array}\right) k^{\underline{m}} V_{k}^{2 m}=n^{\underline{m}}\left(\sum_{i=0}^{m-1}(-1)^{n(i+1)}\left(\begin{array}{c}
2 m \\
i
\end{array}\right) V_{m-i}^{n-m} V_{(m+n)(m-i)}+\left(\begin{array}{c}
2 m \\
m
\end{array}\right) 2^{n-m}\right) .
$$

Keywords: Generalized Fibonacci numbers; sums; falling factorials.

AMS Subject Classification (2020): Primary: 05A10 ; Secondary: 11B37; $11 B 39$.

${ }^{*}$ Corresponding author
\end{abstract}

\section{Introduction}

For $n \geq 2$, define second order linear sequences $\left\{U_{n}\right\}$ and $\left\{V_{n}\right\}$ by

$$
U_{n}=p U_{n-1}+U_{n-2} \text { and } V_{n}=p V_{n-1}+V_{n-2},
$$

with $U_{0}=0, U_{1}=1$ and $V_{0}=2, V_{1}=p$, respectively. When $p=1, U_{n}=F_{n}$ ( $n$th Fibonacci number) and $V_{n}=L_{n}$ ( $n$th Lucas number). The Binet formulae are

$$
U_{n}=\frac{\alpha^{n}-\beta^{n}}{\alpha-\beta} \text { and } V_{n}=\alpha^{n}+\beta^{n},
$$

where $\alpha, \beta=(p \mp \sqrt{\Delta}) / 2$ such that $\Delta=p^{2}+4$.

$x^{\underline{m}}$ stands for the falling factorial defined by

$$
x^{\underline{\underline{m}}}=x(x-1) \ldots(x-m+1) .
$$

Wiemann and Cooper [11] gave certain conjectures for the Melham sum

$$
\sum_{k=0}^{n} F_{2 k+\delta}^{2 m+\varepsilon}
$$

Received : 23-03-2020, Accepted : 05-05-2020 
where $m$ is a positive integer and $\varepsilon, \delta \in\{0,1\}$. Ozeki [9] considered Melham's sum and gave an explicit expansion for it as a polynomial in $F_{2 n+1}$ and Prodinger [10] obtained a formula for the sum

$$
\sum_{k=0}^{n} F_{2 k+\delta}^{2 m+\varepsilon}
$$

where $m, \varepsilon$ and $\delta$ are as before. Kılıç et. al [2] considered alternating Melham's sums for Fibonacci and Lucas numbers of the form

$$
\sum_{k=1}^{n}(-1)^{k} F_{2 k+\delta}^{2 m+\varepsilon} \text { and } \sum_{k=1}^{n}(-1)^{k} L_{2 k+\delta}^{2 m+\varepsilon},
$$

where $m, \varepsilon$ and $\delta$ are as before.

Kılıç and Taşdemir [7] derived various binomial-double-sums involving the Fibonacci numbers as well as their alternating analogues and they [8] gave and computed various sum families of binomial sums namely binomial-sums including double sums and one binomial coefficient of the forms and their alternating analogues

$$
\sum_{0 \leq i, j \leq n}\left(\begin{array}{l}
i \\
j
\end{array}\right) U_{r i+t j}, \quad \sum_{0 \leq i, j \leq n}\left(\begin{array}{l}
i \\
j
\end{array}\right)(-1)^{i} V_{r i+t j},
$$

where $r$ and $t$ are odd integers.

Khan and Kwong [6] obtained the sums

$$
\sum_{i=0}^{n}\left(\begin{array}{c}
n \\
i
\end{array}\right) i^{m} U_{i} \text { and } \sum_{i=0}^{n}\left(\begin{array}{c}
n \\
i
\end{array}\right)(-1)^{i} i^{m} U_{i}
$$

for some nonnegative integer $m$.

Kılıç et. al [3] computed the weighted binomial sums including the powers of the summation index:

$$
\sum_{i=0}^{n}\left(\begin{array}{c}
n \\
i
\end{array}\right) i^{m} U_{t i}^{2 m+\varepsilon} \text { and } \sum_{i=0}^{n}\left(\begin{array}{c}
n \\
i
\end{array}\right) i^{m}(-1)^{n+i} V_{t i}^{2 m+\varepsilon},
$$

where positive integers $t, m$ and $\varepsilon \in\{0,1\}$.

K1liç et. al [4] introduced and computed new kinds of binomial sums including falling factorial of the summation indice. For example,

$$
\begin{aligned}
\sum_{i=0}^{n}\left(\begin{array}{c}
n \\
i
\end{array}\right)(-1)^{i} i^{\underline{m}} U_{k t i} V_{k(n-t i)}= & (-1)^{k n(t+1)+m} n^{\underline{m}} U_{k t}^{n-m} \Delta^{(n-m) / 2} \\
& \times\left\{\begin{array}{cc}
U_{k(t n+t m-n)} & \text { if } n \equiv m \\
-\Delta^{1 / 2} V_{k(t n+t m-n)} & \text { if } n \equiv m+1 \quad(\bmod 2),
\end{array}\right.
\end{aligned}
$$

where $k$ and $t$ are any integers, and $m$ is a nonnegative integer. They [5] gave closed formulæ for weighted and alternating weighted binomial sums with the generalized Fibonacci and Lucas numbers including both falling factorials and powers of indices. Furthermore they derived closed formulæ for weighted binomial sums including odd powers of the generalized Fibonacci and Lucas numbers.

\section{Some Results}

In this section, firstly, we will continue our work with the following lemma:

Lemma 2.1. [1]Let $n$ and $m$ be integers such that $0 \leq m<n$. For $a \neq-1$,

$$
\sum_{k=0}^{n}\left(\begin{array}{l}
n \\
k
\end{array}\right) k^{\underline{m}} a^{k}=a^{m} n^{\underline{m}}(1+a)^{n-m} .
$$


Now, we shall present one of our main result.

Theorem 2.1. For integers $n$ and $m$ such that $0 \leq m<n$, we have

$$
\sum_{k=0}^{n}\left(\begin{array}{l}
n \\
k
\end{array}\right) k^{\underline{m}} U_{2 k}^{2 m}=\frac{n^{\underline{m}}}{\Delta^{m}}\left(\sum_{i=0}^{m}(-1)^{i}\left(\begin{array}{c}
2 m \\
i
\end{array}\right) V_{2(m-i)}^{n-m} V_{2(m+n)(m-i)}-(-1)^{m} 2^{n-m}\left(\begin{array}{c}
2 m \\
m
\end{array}\right)\right)
$$

and

$$
\begin{aligned}
& \sum_{k=0}^{n}\left(\begin{array}{l}
n \\
k
\end{array}\right) k^{\underline{m}} U_{2 k}^{2 m+1}=n^{\underline{m}} \Delta^{(n-3 m) / 2} \\
& \times\left\{\begin{array}{c}
\sum_{i=0}^{m}(-1)^{i}\left(\begin{array}{c}
2 m+1 \\
i
\end{array}\right) U_{2 m-2 i+1}^{n-m} U_{(2 m-2 i+1)(m+n)} \\
\Delta^{-1 / 2} \sum_{i=0}^{m}(-1)^{i}\left(\begin{array}{c}
2 m+1 \\
i
\end{array}\right) U_{2 m-2 i+1}^{n-m} V_{(2 m-2 i+1)(m+n)}
\end{array} \quad \text { if } n \equiv m+1 \quad(\bmod 2) .\right.
\end{aligned}
$$

Proof. Consider that

$$
\begin{aligned}
& \sum_{k=0}^{n}\left(\begin{array}{l}
n \\
k
\end{array}\right) k^{\underline{m}} U_{2 k}^{2 m}=\frac{1}{(\alpha-\beta)^{2 m}} \sum_{k=0}^{n}\left(\begin{array}{c}
n \\
k
\end{array}\right) k^{\underline{m}}\left(\alpha^{2 k}-\beta^{2 k}\right)^{2 m} \\
= & \frac{1}{(\alpha-\beta)^{2 m}} \sum_{k=0}^{n}\left(\begin{array}{l}
n \\
k
\end{array}\right) k^{\underline{m}} \sum_{i=0}^{2 m}(-1)^{i}\left(\begin{array}{c}
2 m \\
i
\end{array}\right) \alpha^{2 k i} \beta^{2 k(2 m-i)} \\
= & \frac{1}{(\alpha-\beta)^{2 m}} \sum_{k=0}^{n}\left(\begin{array}{l}
n \\
k
\end{array}\right) k^{\underline{m}}\left(\sum_{i=0}^{m}(-1)^{i}\left(\begin{array}{c}
2 m \\
i
\end{array}\right)\left(\alpha^{2 k i} \beta^{2 k(2 m-i)}+\alpha^{2 k(2 m-i)} \beta^{2 k i}\right)-(-1)^{m}\left(\begin{array}{c}
2 m \\
m
\end{array}\right)(\alpha \beta)^{2 k m}\right),
\end{aligned}
$$

and by $\alpha \beta=-1$ and Lemma 2.1, the above expression equals

$$
\begin{gathered}
=\frac{1}{(\alpha-\beta)^{2 m}} \sum_{i=0}^{m}(-1)^{i}\left(\begin{array}{c}
2 m \\
i
\end{array}\right) \sum_{k=0}^{n}\left(\begin{array}{l}
n \\
k
\end{array}\right) k^{\underline{m}}\left(\alpha^{2 k(2 m-2 i)}+\beta^{2 k(2 m-2 i)}\right) \\
-(-1)^{m} \frac{1}{(\alpha-\beta)^{2 m}}\left(\begin{array}{c}
2 m \\
m
\end{array}\right) \sum_{k=0}^{n}\left(\begin{array}{l}
n \\
k
\end{array}\right) k^{\underline{m}}(\alpha \beta)^{2 k m} \\
=\frac{1}{(\alpha-\beta)^{2 m}} \sum_{i=0}^{m}(-1)^{i}\left(\begin{array}{c}
2 m \\
i
\end{array}\right) \sum_{k=0}^{n}\left(\begin{array}{l}
n \\
k
\end{array}\right) k^{\underline{m}}\left(\alpha^{4 k(m-i)}+\beta^{4 k(m-i)}\right) \\
-(-1)^{m} \frac{1}{(\alpha-\beta)^{2 m}}\left(\begin{array}{c}
2 m \\
m
\end{array}\right) \sum_{k=0}^{n}\left(\begin{array}{l}
n \\
k
\end{array}\right) k^{\underline{m}} \\
=\frac{n^{\underline{m}}}{\Delta^{m}} \sum_{i=0}^{m}(-1)^{i}\left(\begin{array}{c}
2 m \\
i
\end{array}\right)\left(\begin{array}{c}
\alpha^{4 m}(m-i) \\
\left(1+\alpha^{4(m-i)}\right)^{n-m}+\beta^{4 m(m-i)}\left(1+\beta^{4(m-i)}\right)^{n-m}
\end{array}\right) \\
-(-1)^{m}\left(\begin{array}{c}
2 m \\
m
\end{array}\right) n^{\underline{m}} \frac{2^{n-m}}{\Delta^{m}} .
\end{gathered}
$$


Since $\alpha^{2}=\beta^{-2}$ and $\beta^{2}=\alpha^{-2}$, we write

$$
\begin{aligned}
& \sum_{k=0}^{n}\left(\begin{array}{l}
n \\
k
\end{array}\right) k^{\underline{m}} U_{2 k}^{2 m}=\frac{n^{\underline{m}}}{\Delta^{m}} \sum_{i=0}^{m}(-1)^{i}\left(\begin{array}{c}
2 m \\
i
\end{array}\right) \\
& \times\left(\alpha^{4 m(m-i)}\left(1+\frac{\alpha^{2(m-i)}}{\beta^{2(m-i)}}\right)^{n-m}+\beta^{4 m(m-i)}\left(1+\frac{\beta^{2(m-i)}}{\alpha^{2(m-i)}}\right)^{n-m}\right)-(-1)^{m} \frac{n^{\underline{m}}}{\Delta^{m}} 2^{n-m}\left(\begin{array}{c}
2 m \\
m
\end{array}\right) \\
= & \frac{n^{\underline{m}}}{\Delta^{m}} \sum_{i=0}^{m}(-1)^{i}\left(\begin{array}{c}
2 m \\
i
\end{array}\right) V_{2(m-i)}^{n-m}\left(\alpha^{4 m(m-i)} \beta^{2(m-i)(m-n)}+\alpha^{2(m-i)(m-n)} \beta^{4 m(m-i)}\right) \\
= & \frac{n^{\underline{m}}}{\Delta^{m}}\left(\sum_{i=0}^{m}(-1)^{i}\left(\begin{array}{c}
2 m \\
i
\end{array}\right) V_{2(m-i)}^{n-m}\left(\beta^{-2(m+n)(m-i)}+\alpha^{-2(m+n)(m-i)}\right)-(-1)^{m} 2^{n-m}\left(\begin{array}{c}
2 m \\
m
\end{array}\right)\right) \\
= & \frac{n^{\underline{m}}}{\Delta^{m}}\left(\sum_{i=0}^{m}(-1)^{i}\left(\begin{array}{c}
2 m \\
i
\end{array}\right) V_{2(m-i)}^{n-m} V_{2(m+n)(m-i)}-(-1)^{m} 2^{n-m}\left(\begin{array}{c}
2 m \\
m
\end{array}\right)\right)
\end{aligned}
$$

as claimed. The proof of the binomial sum of the odd powers can be similarly done.

For example, if we take $m=1$,

$$
\sum_{k=0}^{n} k\left(\begin{array}{l}
n \\
k
\end{array}\right) U_{2 k}^{2}=\frac{n}{\Delta}\left((\Delta-2)^{n-1} V_{2(n+1)}-2^{n}\right)
$$

and

$$
\sum_{k=0}^{n}\left(\begin{array}{l}
n \\
k
\end{array}\right) k U_{2 k}^{3}=n \Delta^{(n-3) / 2}\left\{\begin{array}{cc}
(\Delta-3)^{n-1} U_{3(n+1)}-3 U_{n+1} & \text { if } n \text { is odd } \\
\Delta^{-1 / 2}\left((\Delta-3)^{n-1} V_{3(n+1)}-3 V_{n+1}\right) & \text { if } n \text { is even. }
\end{array}\right.
$$

Theorem 2.2. Let $n$ and $m$ be integers such that $0<m<n$. For even number $m$,

$$
\sum_{k=0}^{n}\left(\begin{array}{l}
n \\
k
\end{array}\right) k^{\underline{m}} V_{k}^{2 m}=n^{\underline{m}}\left(\sum_{i=0}^{m-1}(-1)^{n i}\left(\begin{array}{c}
2 m \\
i
\end{array}\right) V_{m-i}^{n-m} V_{(m+n)(m-i)}+(-1)^{m}\left(\begin{array}{c}
2 m \\
m
\end{array}\right) 2^{n-m}\right),
$$

and for odd number $m$,

$$
\sum_{k=0}^{n}\left(\begin{array}{l}
n \\
k
\end{array}\right) k^{\underline{m}} V_{k}^{2 m}=n^{\underline{m}} \Delta^{(n-m) / 2}\left\{\begin{array}{cl}
\Delta^{1 / 2} \sum_{i=0}^{m-1}(-1)^{n i}\left(\begin{array}{c}
2 m \\
i
\end{array}\right) U_{m-i}^{n-m} U_{(m+n)(m-i)} & \text { if } n \text { is even, } \\
\sum_{i=0}^{m-1}(-1)^{n i}\left(\begin{array}{c}
2 m \\
i
\end{array}\right) U_{m-i}^{n-m} V_{(m+n)(m-i)} & \text { if } n \text { is odd. }
\end{array}\right.
$$

Theorem 2.3. Let $n$ and $m$ be integers such that $0 \leq m<n$. We have

$$
\sum_{k=0}^{n}\left(\begin{array}{l}
n \\
k
\end{array}\right) k^{\underline{m}} V_{2 k}^{2 m+1}=n^{\underline{m}} \Delta^{(n-m) / 2}\left\{\begin{array}{cc}
\sum_{i=0}^{m}\left(\begin{array}{c}
2 m+1 \\
i
\end{array}\right) U_{2 m-2 i+1}^{n-m} V_{(2 m-2 i+1)(m+n)} & \text { if } n \equiv m \quad(\bmod 2), \\
\Delta^{1 / 2} \sum_{i=0}^{m}\left(\begin{array}{c}
2 m+1 \\
i
\end{array}\right) U_{2 m-2 i+1}^{n-m} U_{(2 m-2 i+1)(m+n)} & \text { if } n \equiv m+1 \quad(\bmod 2),
\end{array}\right.
$$

and

$$
\sum_{k=0}^{n}\left(\begin{array}{l}
n \\
k
\end{array}\right) k^{\underline{m}} V_{2 k}^{2 m}=n^{\underline{m}}\left(\sum_{i=0}^{m-1}\left(\begin{array}{c}
2 m \\
i
\end{array}\right) V_{2(m-i)}^{n-m} V_{2(m+n)(m-i)}+\left(\begin{array}{c}
2 m \\
m
\end{array}\right) 2^{n-m}\right) .
$$

Now, we will give alternating binomial sums of the powers of generalized Fibonacci and Lucas numbers with falling factorials. 
Theorem 2.4. Let $n$ and $m$ be integers such that $0 \leq m<n$. We have

$$
\begin{aligned}
& \sum_{k=0}^{n}(-1)^{k}\left(\begin{array}{l}
n \\
k
\end{array}\right) k^{\underline{m}} U_{2 k}^{2 m}=(-1)^{m} n^{\underline{m}} \Delta^{(n-3 m) / 2} \\
& \times\left\{\begin{array}{cc}
\sum_{i=0}^{m}(-1)^{i}\left(\begin{array}{c}
2 m \\
i
\end{array}\right) U_{2(m-i)}^{n-m} V_{2(m+n)(m-i)} & \text { if } n \equiv m \quad(\bmod 2), \\
\Delta^{1 / 2} \sum_{i=0}^{m}(-1)^{i+1}\left(\begin{array}{c}
2 m \\
i
\end{array}\right) U_{2(m-i)}^{n-m} U_{2(m+n)(m-i)} & \text { if } n \equiv m+1 \quad(\bmod 2),
\end{array}\right.
\end{aligned}
$$

and

$$
\sum_{k=0}^{n}(-1)^{k}\left(\begin{array}{l}
n \\
k
\end{array}\right) k^{\underline{m}} U_{2 k}^{2 m+1}=\frac{(-1)^{n}}{\Delta^{m}} n^{\underline{m}} \sum_{i=0}^{m}(-1)^{i}\left(\begin{array}{c}
2 m+1 \\
i
\end{array}\right) V_{2 m-2 i+1}^{n-m} U_{(2 m+1-2 i)(m+n)} .
$$

Proof. From Binet formula of $\left\{U_{n}\right\}$, we have

$$
\begin{aligned}
& \sum_{k=0}^{n}(-1)^{k}\left(\begin{array}{l}
n \\
k
\end{array}\right) k^{\underline{m}} U_{2 k}^{2 m}=\frac{1}{(\alpha-\beta)^{2 m}} \sum_{k=0}^{n}(-1)^{k}\left(\begin{array}{l}
n \\
k
\end{array}\right) k^{\underline{m}} \\
& \times\left(\sum_{i=0}^{m}(-1)^{i}\left(\begin{array}{c}
2 m \\
i
\end{array}\right)\left(\alpha^{2 k i} \beta^{2 k(2 m-i)}+\alpha^{2 k(2 m-i)} \beta^{2 k i}\right)-(-1)^{m}\left(\begin{array}{c}
2 m \\
m
\end{array}\right)(\alpha \beta)^{2 k m}\right),
\end{aligned}
$$

and applying some elementary operations, equals

$$
\begin{aligned}
=\frac{1}{(\alpha-\beta)^{2 m}} \sum_{i=0}^{m}( & -1)^{i}\left(\begin{array}{c}
2 m \\
i
\end{array}\right) \sum_{k=0}^{n}(-1)^{k}\left(\begin{array}{l}
n \\
k
\end{array}\right) k^{\underline{m}}\left(\alpha^{4 k(m-i)}+\beta^{4 k(m-i)}\right) \\
& -(-1)^{m}\left(\begin{array}{c}
2 m \\
m
\end{array}\right) \frac{1}{(\alpha-\beta)^{2 m}} \sum_{k=0}^{n}\left(\begin{array}{l}
n \\
k
\end{array}\right) k^{\underline{m}}(-1)^{k}(\alpha \beta)^{2 k m} .
\end{aligned}
$$

By Lemma 2.1 and $\alpha \beta=-1$, we have

$$
\begin{aligned}
= & \frac{1}{(\alpha-\beta)^{2 m}} \sum_{i=0}^{m}(-1)^{i}\left(\begin{array}{c}
2 m \\
i
\end{array}\right) \sum_{k=0}^{n}(-1)^{k}\left(\begin{array}{l}
n \\
k
\end{array}\right) k^{\underline{m}}\left(\alpha^{4 k(m-i)}+\beta^{4 k(m-i)}\right) \\
& \quad-(-1)^{m}\left(\begin{array}{c}
2 m \\
m
\end{array}\right) \frac{1}{(\alpha-\beta)^{2 m}} \sum_{k=0}^{n}(-1)^{k}\left(\begin{array}{c}
n \\
k
\end{array}\right) k^{\underline{m}} \\
= & \frac{n^{\underline{m}}}{\Delta^{m}} \sum_{i=0}^{m}(-1)^{i+m}\left(\begin{array}{c}
2 m \\
i
\end{array}\right)\left(\alpha^{4 m(m-i)}\left(1-\alpha^{4(m-i)}\right)^{n-m}+\beta^{4 m(m-i)}\left(1-\beta^{4(m-i)}\right)^{n-m}\right) \\
= & (-1)^{m} \frac{n^{\underline{m}}}{\Delta^{m}} \sum_{i=0}^{m}(-1)^{i}\left(\begin{array}{c}
2 m \\
i
\end{array}\right)\left(\alpha^{4 m(m-i)}\left(1-\frac{\alpha^{2(m-i)}}{\beta^{2(m-i)}}\right)^{n-m}+\beta^{4 m(m-i)}\left(1-\frac{\beta^{2(m-i)}}{\alpha^{2(m-i)}}\right)^{n-m}\right) \\
= & (-1)^{m} \frac{n^{\underline{m}}}{\Delta^{m}}(\alpha-\beta)^{n-m} \sum_{i=0}^{m}(-1)^{i}\left(\begin{array}{c}
2 m \\
i
\end{array}\right) U_{2(m-i)}^{n-m}\left((-1)^{n-m} \alpha^{2(m+n)(m-i)}+\beta^{2(m+n)(m-i)}\right) .
\end{aligned}
$$

For $n \equiv m(\bmod 2)$, we get

$$
\sum_{k=0}^{n}(-1)^{k}\left(\begin{array}{l}
n \\
k
\end{array}\right) k^{\underline{m}} U_{2 k}^{2 m}=(-1)^{m} \Delta^{(n-3 m) / 2} n^{\underline{m}} \sum_{i=0}^{m}(-1)^{i}\left(\begin{array}{c}
2 m \\
i
\end{array}\right) U_{2(m-i)}^{n-m} V_{2(m+n)(m-i)} .
$$

For $n \equiv m+1(\bmod 2)$, the desired result is similarly obtained. We can prove the other claim by using same approach.

For positive integer $n>1$ and $m=1$,

$$
\sum_{k=0}^{n}(-1)^{k}\left(\begin{array}{l}
n \\
k
\end{array}\right) k U_{2 k}^{2}=\left\{\begin{array}{cc}
-n \Delta^{(n-3) / 2} U_{2}^{n-1} V_{2(n+1)} & \text { if } n \text { is odd } \\
n \Delta^{(n-2) / 2} U_{2}^{n-1} U_{2(n+1)} & \text { if } n \text { is even }
\end{array}\right.
$$


and for $m=2$,

$$
\begin{aligned}
& \sum_{k=0}^{n}(-1)^{k}\left(\begin{array}{l}
n \\
k
\end{array}\right) k(k-1) U_{2 k}^{5} \\
& =(-1)^{n} p^{n-2} \frac{n(n-1)}{\Delta^{2}}\left(\left(p^{4}+5 p^{2}+5\right)^{n-2} U_{5(n+2)}-5\left(p^{2}+3\right)^{n-2} U_{3(n+2)}+10 U_{(n+2)}\right) .
\end{aligned}
$$

Theorem 2.5. Let $n$ and $m$ be integers such that $0<m<n$. For even number $m$,

$$
\sum_{k=0}^{n}(-1)^{k}\left(\begin{array}{l}
n \\
k
\end{array}\right) k^{\underline{m}} V_{k}^{2 m}=n^{\underline{m}} \Delta^{(n-m) / 2}\left\{\begin{array}{cc}
\sum_{i=0}^{m-1}\left(\begin{array}{c}
2 m \\
i
\end{array}\right) U_{m-i}^{n-m} V_{(m+n)(m-i)} & \text { if } n \text { is even, } \\
\Delta^{1 / 2} \sum_{i=0}^{m-1}(-1)^{i+1}\left(\begin{array}{c}
2 m \\
i
\end{array}\right) U_{m-i}^{n-m} U_{(m+n)(m-i)} & \text { if } n \text { is odd, }
\end{array}\right.
$$

and for odd number $m$,

$$
\sum_{k=0}^{n}(-1)^{k}\left(\begin{array}{l}
n \\
k
\end{array}\right) k^{\underline{m}} V_{k}^{2 m}=n^{\underline{m}}\left(\sum_{i=0}^{m-1}(-1)^{n(i+1)}\left(\begin{array}{c}
2 m \\
i
\end{array}\right) V_{m-i}^{n-m} V_{(m+n)(m-i)}+\left(\begin{array}{c}
2 m \\
m
\end{array}\right) 2^{n-m}\right) .
$$

Theorem 2.6. Let $n$ and $m$ be integers such that $0<m<n$. We have

$$
\sum_{k=0}^{n}(-1)^{k}\left(\begin{array}{l}
n \\
k
\end{array}\right) k^{\underline{m}} V_{2 k}^{2 m+1}=(-1)^{n} n^{\underline{m}} \sum_{i=0}^{m}\left(\begin{array}{c}
2 m+1 \\
i
\end{array}\right) V_{2 m-2 i+1}^{n-m} V_{(2 m-2 i+1)(m+n)},
$$

and

$$
\sum_{k=0}^{n}(-1)^{k}\left(\begin{array}{l}
n \\
k
\end{array}\right) k^{\underline{m}} V_{2 k}^{2 m}=(-1)^{m} n^{\underline{m}} \Delta^{(n-m) / 2}\left\{\begin{array}{cc}
\sum_{i=0}^{m-1}\left(\begin{array}{c}
2 m \\
i
\end{array}\right) U_{2(m-i)}^{n-m} V_{2(m+n)(m-i)} & \text { if } n \equiv m \quad(\bmod 2), \\
\Delta^{1 / 2} \sum_{i=0}^{m-1}\left(\begin{array}{c}
2 m \\
i
\end{array}\right) U_{2(m-i)}^{n-m} U_{2(m+n)(m-i)} & \text { if } n \equiv m+1 \quad(\bmod 2) .
\end{array}\right.
$$

\section{References}

[1] Gould, H.W.: Combinatorial identities. Morgantown. W. Va. (1972).

[2] Kılıç, E., Ömür, N. and Türker Ulutaş, Y.: Alternating sums of the powers of Fibonacci and Lucas numbers. Miskolc Mathematical Notes. 12 (1), 87-103 (2011).

[3] Kılıç, E., Türker Ulutaş, Y. and Ömür, N.: Formulas for weighted binomial sums with the powers of terms of binary recurrences. Miskolc Mathematical Notes. 13 (1), 53-65 (2012).

[4] Kılıç, E., Ömür, N. and Koparal, S.: On alternating weighted binomial sums with falling factorials. Bulletin of Mathematical Analysis and Applications. 9 (1), 58-64 (2017).

[5] Kılıç, E., Ömür, N. and Koparal, S.: Formulae for two weighted binomial identities with the falling factorials. Ars Combinatoria. 138, 223-231 (2018).

[6] Khan, M. and Kwong, H.: Some binomial identities associated with the generalized natural number sequence. The Fibonacci Quarterly. 49 (1), 57-65 (2011).

[7] K1lıç, E. and Taşdemir, F.: On binomial double sums with Fibonacci and Lucas Numbers-I. Ars Combinatoria. 144, 173-185 (2019).

[8] Kılıç, E. and Taşdemir, F.: On binomial double sums with Fibonacci and Lucas Numbers-II. Ars Combinatoria. 144, 345-354 (2019).

[9] Ozeki, K.: On Melham's sum. Fibonacci Quarterly. 46/47 (2), 107-110 (2008/09).

[10] Prodinger, H.: On a sum of Melham and its variants. Fibonacci Quarterly. 46/47 (3), 207-215 (2008/09).

[11] Wiemann, M. and Cooper, C.: Divisibility of an F-L type convolution, in Applications of Fibonacci numbers. ser. Proceedings of the 10th International Research Conference on Fibonacci Numbers and their Applications held at Northern Arizona University, Dorrecht: Klywer Acad. Publ. 9, 267-287 (2004). 


\section{Affiliations}

\section{SIBEL KOPARAL}

AdDRESS: Kocaeli University, Department of Mathematics, 41380, Kocaeli-Turkey. E-MAIL: sibel.koparal@kocaeli.edu.tr

\section{ORCID ID:0000-0001-9574-9652}

\section{NEŞE ÖMÜR}

ADDRESS: Kocaeli University, Department of Mathematics, 41380, Kocaeli-Turkey.

E-MAIL: neseomur@kocaeli.edu.tr

ORCID ID:0000-0002-3972-9910 